\title{
SPIN MODELS CONSTRUCTED FROM HADAMARD MATRICES
}

\author{
TAKUYA IKUTA AND AKIHIRO MUNEMASA
}

\begin{abstract}
A spin model (for link invariants) is a square matrix $W$ which satisfies certain axioms. For a spin model $W$, it is known that $W^{T} W^{-1}$ is a permutation matrix, and its order is called the index of $W$. F. Jaeger and K. Nomura found spin models of index 2, by modifying the construction of symmetric spin models from Hadamard matrices.

The aim of this paper is to give a construction of spin models of an arbitrary even index from any Hadamard matrix. In particular, we show that our spin models of indices a power of 2 are new.
\end{abstract}

\section{INTRODUCTION}

The notion of spin model was introduced by V.F.R. Jones 13 to construct invariants of knots and links. The original definition due to Jones requires that a spin model be a symmetric matrix, but later by K. Kawagoe, A. Munemasa, and Y. Watatani 14, a general definition allowing non-symmetric matrices is given. In this paper, we consider spin models which are not necessarily symmetric.

Let $X$ be a non-empty finite set. We denote by $\operatorname{Mat}_{X}\left(\mathbb{C}^{*}\right)$ the set of square matrices with non-zero complex entries whose rows and columns are indexed by $X$. For $W \in \operatorname{Mat}_{X}\left(\mathbb{C}^{*}\right)$ and $x, y \in X$, the $(x, y)$-entry of $W$ is denoted by $W(x, y)$. A spin model $W \in \operatorname{Mat}_{X}\left(\mathbb{C}^{*}\right)$ is defined to be a matrix which satisfies two conditions (type II and type III; see Section 2).

One of the examples of spin models is a Potts model, defined as follows. Let $X$ be a finite set with $r$ elements, and let $I, J \in \operatorname{Mat}_{X}\left(\mathbb{C}^{*}\right)$ be the identity matrix and the all 1's matrix, respectively. Let $u$ be a complex number satisfying

$$
\begin{aligned}
\left(u^{2}+u^{-2}\right)^{2} & =r \text { if } r \geq 2, \\
u^{4} & =1 \text { if } r=1 .
\end{aligned}
$$

Then a Potts model $A_{u}$ is defined as

$$
A_{u}=u^{3} I-u^{-1}(J-I) .
$$

As examples of spin models, we know only Potts models [13, 10], spin models on finite abelian groups [3, 5, Jaeger's Higman-Sims model 10, Hadamard models [17, 12, non-symmetric Hadamard models [12, and tensor products of these. Apart from spin models on finite abelian groups, non-symmetric Hadamard models are essentially the only known family of non-symmetric spin models.

If $W$ is a spin model, then by [12, Proposition 2], $R=W^{T} W^{-1}$ is a permutation matrix. The order of $R$ as a permutation is called the index of the spin model $W$.

Date: January 6, 2011.

2000 Mathematics Subject Classification. 05B20,05E30.

Key words and phrases. spin model, association scheme, Hadamard matrix. 
A Hadamard matrix of order $r$ is a square matrix $H$ of size $r$ with entries \pm 1 satisfying $H H^{T}=I$. In [12, F. Jaeger and K. Nomura constructed non-symmetric Hadamard models, which are spin models of index 2:

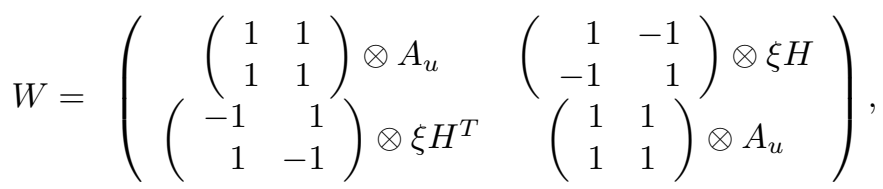

where $\xi$ is a primitive 8-th root of unity, $A_{u} \in \operatorname{Mat}_{X}\left(\mathbb{C}^{*}\right)$ is a Potts model, and $H \in \operatorname{Mat}_{X}\left(\mathbb{C}^{*}\right)$ is a Hadamard matrix.

Note that non-symmetric Hadamard models are a modification of the earlier Hadamard models ([12, see also [12, Section 5]), defined by

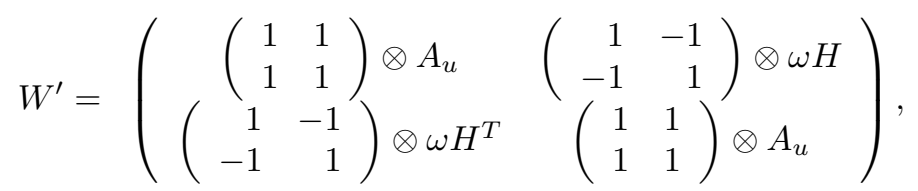

where $\omega$ is a 4 -th root of unity.

To construct spin models of index $m>2$, it seems natural to consider an $m \times m$ block matrix $W=\left(W_{i, j}\right)_{i, j \in \mathbb{Z}_{m}}$ such that each block $W_{i j}$ is the tensor product of two matrices like those in (2) and (3):

$$
W_{i j}=S_{i j} \otimes T_{i j} \quad\left(i, j \in \mathbb{Z}_{m}\right) .
$$

Such matrices appeared in [9, Proposition 6.2], with the matrices $S_{i j} \in M_{a} t_{\mathbb{Z}_{m}}\left(\mathbb{C}^{*}\right)$ given by

$$
S_{i j}\left(\ell, \ell^{\prime}\right)=\eta^{\left(\ell-\ell^{\prime}\right)(i-j)} \quad\left(\ell, \ell^{\prime} \in \mathbb{Z}_{m}\right),
$$

where $\eta$ is a primitive $m$-th root of unity.

In this paper, we construct an infinite class of spin models of even index containing non-symmetric Hadamard models. Also, we construct an infinite class of symmetric spin models containing Hadamard models. Our main result is as follows:

Theorem 1.1. Let $r$ be a positive integer, and let $m$ be an even positive integer. Define $Y=\{1, \ldots, r\}, X_{i}=\left\{(i, \ell, x) \mid \ell \in \mathbb{Z}_{m}, x \in Y\right\}$ for $i \in \mathbb{Z}_{m}$, and $X=$ $X_{0} \cup \cdots \cup X_{m-1}$. Let $A_{u}, H \in M t_{Y}\left(\mathbb{C}^{*}\right)$ be a Potts model and a Hadamard matrix, respectively. Define $V_{i j}$ for $i, j \in \mathbb{Z}_{m}$ by

$$
V_{i j}= \begin{cases}A_{u} & \text { if } i-j \text { is even, } \\ H & \text { if }(i, j) \equiv(0,1) \quad(\bmod 2), \\ H^{T} & \text { if }(i, j) \equiv(1,0) \quad(\bmod 2) .\end{cases}
$$

Then the following statements hold:

(i) Let a be a primitive $2 m^{2}$-th root of unity. Let $W \in M a t_{X}\left(\mathbb{C}^{*}\right)$ be the matrix whose $(\alpha, \beta)$ entry is given by $a^{2 m\left(\ell-\ell^{\prime}\right)(i-j)+\epsilon(i, j)} V_{i j}(x, y)$ for $\alpha=$ $(i, \ell, x), \beta=\left(j, \ell^{\prime}, y\right) \in X$, where $\epsilon(i, j)=(i-j)^{2}+m(i-j)$. Then $W$ is a spin model of index $m$.

(ii) Let $\eta$ be a primitive $m$-th root of unity, and let $b$ be an $m^{2}$-th root of unity. Let $W^{\prime} \in \operatorname{Mat}_{X}\left(\mathbb{C}^{*}\right)$ be the matrix whose $(\alpha, \beta)$ entry is given by $\eta^{\left(\ell-\ell^{\prime}\right)(i-j)} b^{\delta(i, j)} V_{i j}$ for $\alpha=(i, \ell, x), \beta=\left(j, \ell^{\prime}, y\right) \in X$, where $\delta(i, j)=$ $(i-j)^{2}$. Then $W^{\prime}$ is a symmetric spin model. 
Note that, in order for $a^{\epsilon(i, j)}$ and $b^{\delta(i, j)}$ to be well-defined, we need to identify $\mathbb{Z}_{m}$ with the subset $\{0,1, \ldots, m-1\}$ of integers.

Remark 1.2. In Theorem 1.1(i), if we define $S_{i j}$ by (5) with $\eta=a^{2 m}$, and $T_{i j}$ by $T_{i j}=a^{\epsilon(i, j)} V_{i j}$, then the $\left(X_{i}, X_{j}\right)$-block of the matrix $W$ is given by (4). Similarly, in Theorem 1.1 (ii), (4) holds with $T_{i j}=b^{\delta(i, j)} V_{i j}$.

The spin models $W, W^{\prime}$ given in Theorem 1.1 are determined by a Hadamard matrix $H$ of order $r$, a complex number $u$ satisfying (11), and a primitive $2 m^{2}$ th root of unity $a$ or an $m^{2}$ th root of unity $b$, respectively. Throughout this paper, we denote by $W_{H, u, a}, W_{H, u, b}^{\prime}$ the spin models given by Theorem1.1(i), (ii), respectively.

Observe that, for any spin models $W_{i}(i=1,2)$ of indices $m_{i}$, their tensor product $W_{1} \otimes W_{2}$ is also a spin model of index $\operatorname{LCM}\left(m_{1}, m_{2}\right)$. In Section 5 , we show that the non-symmetric spin model $W_{H, u, a}$ whose index is a power of 2 is new in the following sense:

Theorem 1.3. Let $H$ be a Hadamard matrix of order $r$. Let $W_{H, u, a}$ be a spin model given in Theorem 1.1 (i), whose index $m$ is a power of 2. If $r>4$, then $W_{H, u, a}$ cannot be decomposed into a tensor product of known spin models.

We note that the list of known spin models is given in Section 5. Jaeger and Nomura [12, p.278] expected that new non-symmetric spin models of index a power of 2 should be found, and our results confirm this expectation.

\section{Type II And Type III CONDITIONS ON BLOCK MATRICES OF TENSOR} PRODUCTS

First we define a spin model. A type II matrix on a finite set $X$ is a matrix $W \in \operatorname{Mat}_{X}\left(\mathbb{C}^{*}\right)$ which satisfies the type II condition:

$$
\sum_{x \in X} \frac{W(\alpha, x)}{W(\beta, x)}=n \delta_{\alpha, \beta} \quad(\text { for all } \alpha, \beta \in X) .
$$

Let $W^{-} \in \operatorname{Mat}_{X}\left(\mathbb{C}^{*}\right)$ be defined by $W^{-}(x, y)=W(y, x)^{-1}$. Then the type II condition is written as $W W^{-}=n I$. Hence, if $W$ is a type II matrix, then $W$ is non-singular with $W^{-1}=n^{-1} W^{-}$.

A type II matrix $W \in \operatorname{Mat}_{X}\left(\mathbb{C}^{*}\right)$ is called a spin model if $W$ satisfies the type III condition:

$$
\sum_{x \in X} \frac{W(\alpha, x) W(\beta, x)}{W(\gamma, x)}=D \frac{W(\alpha, \beta)}{W(\alpha, \gamma) W(\gamma, \beta)} \quad(\text { for all } \alpha, \beta, \gamma \in X)
$$

for some nonzero real number $D$ with $D^{2}=n$, which is independent of the choice of $\alpha, \beta, \gamma \in X$.

Let $m$ be a positive integer. In this section, assuming that $W$ is an $m \times m$ block matrix with blocks of the form (4), we will establish conditions on $T_{i j}$ under which $W$ satisfies the type II and type III conditions. Some parts of these conditions are already given in [9, Proposition 5.1, Proposition 6.2].

Let $\eta$ be a primitive $m$-th root of unity, and let $S_{i j}$ be the matrix of size $m$ defined by (5) for $i, j \in \mathbb{Z}_{m}$. Let $r$ be a positive integer, and define $Y=\{1, \ldots, r\}$, $X_{i}=\left\{(i, \ell, x) \mid \ell \in \mathbb{Z}_{m}, x \in Y\right\}$ for $i \in \mathbb{Z}_{m}$, and $X=X_{0} \cup \cdots \cup X_{m-1}$. Let $T_{i j} \in \operatorname{Mat}_{Y}\left(\mathbb{C}^{*}\right)$ be a matrix for $i, j \in \mathbb{Z}_{m}$, and let $W_{i j}$ be the matrix defined by 
(4). Let $W \in \operatorname{Mat}_{X}\left(\mathbb{C}^{*}\right)$ be the matrix whose $\left(X_{i}, X_{j}\right)$-block is $W_{i j}$ for $i, j \in \mathbb{Z}_{m}$. Then

$$
W\left((i, \ell, x),\left(j, \ell^{\prime}, y\right)\right)=S_{i j}\left(\ell, \ell^{\prime}\right) T_{i j}(x, y) .
$$

Lemma 2.1 ([9, Proposition 5.1]). The matrix $W$ is a type II matrix if and only if $T_{i j}$ is a type II matrix for all $i, j \in \mathbb{Z}_{m}$.

Lemma 2.2. The matrix $W$ satisfies the type III condition (8) if and only if the following equality holds for all $i_{1}, i_{2}, i_{3} \in \mathbb{Z}_{m}$ and $x_{1}, x_{2}, x_{3} \in Y$ :

$$
\sum_{x \in Y} \frac{T_{i_{1}, i_{0}}\left(x_{1}, x\right) T_{i_{2}, i_{0}}\left(x_{2}, x\right)}{T_{i_{3}, i_{0}}\left(x_{3}, x\right)}=\frac{D}{m} \cdot \frac{T_{i_{1}, i_{2}}\left(x_{1}, x_{2}\right)}{T_{i_{1}, i_{3}}\left(x_{1}, x_{3}\right) T_{i_{3}, i_{2}}\left(x_{3}, x_{2}\right)},
$$

where $i_{0}=i_{1}+i_{2}-i_{3} \bmod m$.

Proof. The type III condition (8) for $\alpha=\left(i_{1}, \ell_{1}, x_{1}\right), \beta=\left(i_{2}, \ell_{2}, x_{2}\right), \gamma=\left(i_{3}, \ell_{3}, x_{3}\right)$ is equivalent to

$$
\begin{aligned}
& \sum_{i, \ell \in \mathbb{Z}_{m}} \frac{\eta^{\left(\ell_{1}-\ell\right)\left(i_{1}-i\right)} \eta^{\left(\ell_{2}-\ell\right)\left(i_{2}-i\right)}}{\eta^{\left(\ell_{3}-\ell\right)\left(i_{3}-i\right)}} \sum_{x \in Y} \frac{T_{i_{1}, i}\left(x_{1}, x\right) T_{i_{2}, i}\left(x_{2}, x\right)}{T_{i_{3}, i}\left(x_{3}, x\right)} \\
& =D \frac{\eta^{\left(\ell_{1}-\ell_{2}\right)\left(i_{1}-i_{2}\right)}}{\eta^{\left(\ell_{1}-\ell_{3}\right)\left(i_{1}-i_{3}\right)} \eta^{\left(\ell_{3}-\ell_{2}\right)\left(i_{3}-i_{2}\right)}} \cdot \frac{T_{i_{1}, i_{2}}\left(x_{1}, x_{2}\right)}{T_{i_{1}, i_{3}}\left(x_{1}, x_{3}\right) T_{i_{3}, i_{2}}\left(x_{3}, x_{2}\right)} .
\end{aligned}
$$

By a direct computation, we obtain

$$
\begin{aligned}
& \frac{\eta^{\left(\ell_{1}-\ell\right)\left(i_{1}-i\right)} \eta^{\left(\ell_{2}-\ell\right)\left(i_{2}-i\right)}}{\eta^{-\left(\ell_{3}-\ell\right)\left(i_{3}-i\right)}} \cdot \frac{\eta^{\left(\ell_{1}-\ell_{3}\right)\left(i_{1}-i_{3}\right)} \eta^{\left(\ell_{3}-\ell_{2}\right)\left(i_{3}-i_{2}\right)}}{\eta^{\left(\ell_{1}-\ell_{2}\right)\left(i_{1}-i_{2}\right)}} \\
& =\eta^{\left(\ell_{1}+\ell_{2}-\ell_{3}-\ell\right)\left(i_{1}+i_{2}-i_{3}-i\right)} .
\end{aligned}
$$

So (8) is equivalent to

$$
\begin{aligned}
& \sum_{i \in \mathbb{Z}_{m}}\left(\sum_{\ell \in \mathbb{Z}_{m}} \eta^{\left(\ell_{1}+\ell_{2}-\ell_{3}-\ell\right)\left(i_{1}+i_{2}-i_{3}-i\right)}\right) \sum_{x \in Y} \frac{T_{i_{1}, i}\left(x_{1}, x\right) T_{i_{2}, i}\left(x_{2}, x\right)}{T_{i_{3}, i}\left(x_{3}, x\right)} \\
& =D \frac{T_{i_{1}, i_{2}}\left(x_{1}, x_{2}\right)}{T_{i_{1}, i_{3}}\left(x_{1}, x_{3}\right) T_{i_{3}, i_{2}}\left(x_{3}, x_{2}\right)} .
\end{aligned}
$$

Since $\eta$ is a primitive $m$-th root of unity and $i_{0}=i_{1}+i_{2}-i_{3} \bmod m$, we have

$$
\sum_{\ell \in \mathbb{Z}_{m}} \eta^{\left(\ell_{1}+\ell_{2}-\ell_{3}-\ell\right)\left(i_{1}+i_{2}-i_{3}-i\right)}=m \delta_{i, i_{0}} .
$$

Thus (11) is equivalent to (10).

We remark that in [9, Proposition 6.2] only the necessity of (10) for the type III condition is proved.

Let $z_{m}$ be the permutation matrix of order $m$ :

$$
z_{m}=\left(\begin{array}{cccc}
1 & & & 1 \\
& \ddots & & \\
& & 1
\end{array}\right) .
$$

We define the permutation matrix $R$ of size $n=m^{2} r$ by $R=I_{m} \otimes z_{m} \otimes I_{r}$, where $I_{m}$ and $I_{r}$ are the identity matrices of size $m$ and $r$, respectively. The order of $R$ is $m$. 
Lemma 2.3. The matrix $W$ satisfies $W^{T} W^{-1}=R$ if and only if $T_{i j}=\eta^{i-j} T_{j i}^{T}$ holds for all $i, j \in \mathbb{Z}_{m}$.

Proof. For $\alpha=(i, \ell, x)$ and $\beta=\left(j, \ell^{\prime}, y\right) \in X$,

$$
\begin{aligned}
W^{T}(\alpha, \beta) & =W(\beta, \alpha) \\
& =\eta^{\left(\ell^{\prime}-\ell\right)(j-i)} T_{j, i}(y, x), \\
(R W)(\alpha, \beta) & =\left(\left(I_{m} \otimes z_{m} \otimes I_{r}\right) W\right)\left((i, \ell, x),\left(j, \ell^{\prime}, y\right)\right) \\
& =W\left((i, \ell-1, x),\left(j, \ell^{\prime}, y\right)\right) \\
& =\eta^{\left(\ell-1-\ell^{\prime}\right)(i-j)} T_{i j}(x, y) \\
& =\eta^{\left(\ell^{\prime}-\ell\right)(j-i)} \eta^{-(i-j)} T_{i j}(x, y) .
\end{aligned}
$$

Therefore $R=W^{T} W^{-1}$ if and only if $T_{j i}(y, x)=\eta^{-(i-j)} T_{i j}(x, y)$ holds for all $i, j \in \mathbb{Z}_{m}$ and $x, y \in Y$.

\section{Proof of Theorem 1.1}

From Remark 1.2 the results in Section 2 can be used for the matrices $W$ and $W^{\prime}$ given in Theorem 1.1 if we define $T_{i j}$ according to Remark 1.2.

For a mapping $g$ from $\mathbb{Z}^{2}$ to $\mathbb{Z}$, we denote by $\lambda_{g}$ the mapping from $\mathbb{Z}^{4}$ to $\mathbb{Z}$ defined by

$$
\lambda_{g}\left(i_{1}, i_{2}, i_{3}, i_{4}\right)=g\left(i_{1}, i_{4}\right)+g\left(i_{2}, i_{4}\right)-g\left(i_{3}, i_{4}\right)+g\left(i_{1}, i_{3}\right)+g\left(i_{3}, i_{2}\right)-g\left(i_{1}, i_{2}\right) .
$$

Recall that we regard $\mathbb{Z}_{m}$ as the subset $\{0,1, \ldots, m-1\}$ of $\mathbb{Z}$, and $\delta, \epsilon: \mathbb{Z}^{2} \rightarrow \mathbb{Z}$ are defined by $\delta(i, j)=(i-j)^{2}, \epsilon(i, j)=\delta(i, j)+m(i-j)$, respectively.

Lemma 3.1. For all $i_{1}, i_{2}, i_{3}, i_{4} \in \mathbb{Z}$, we have

$$
\begin{aligned}
& \lambda_{\delta}\left(i_{1}, i_{2}, i_{3}, i_{4}\right)=\left(i_{1}+i_{2}-i_{3}-i_{4}\right)^{2} \\
& \lambda_{\epsilon}\left(i_{1}, i_{2}, i_{3}, i_{4}\right)=\left(i_{1}+i_{2}-i_{3}-i_{4}\right)\left(i_{1}+i_{2}-i_{3}-i_{4}+m\right) .
\end{aligned}
$$

In particular, if $i_{0}=i_{1}+i_{2}-i_{3}(\bmod m)$, then

$$
\begin{aligned}
& \lambda_{\delta}\left(i_{1}, i_{2}, i_{3}, i_{0}\right) \equiv 0 \quad\left(\bmod m^{2}\right) \\
& \lambda_{\epsilon}\left(i_{1}, i_{2}, i_{3}, i_{0}\right) \equiv 0 \quad\left(\bmod 2 m^{2}\right) .
\end{aligned}
$$

Proof. Straightforward.

In [12, $\S 5.1]$, the following is used to construct non-symmetric or symmetric Hadamard models: 
Lemma $3.2([12, \S 5.1])$. Let $A_{u}, H \in \operatorname{Mat}_{Y}\left(\mathbb{C}^{*}\right)$ be a Potts model and a Hadamard matrix, respectively. Then the following holds for all $x_{1}, x_{2}, x_{3} \in Y$ :

$$
\begin{aligned}
\sum_{y \in Y} \frac{A_{u}\left(x_{1}, y\right) A_{u}\left(x_{2}, y\right)}{A_{u}\left(x_{3}, y\right)} & =D_{u} \frac{A_{u}\left(x_{1}, x_{2}\right)}{A_{u}\left(x_{1}, x_{3}\right) A_{u}\left(x_{3}, x_{2}\right)}, \\
\sum_{y \in Y} A_{u}\left(x_{1}, y\right) H\left(y, x_{2}\right) H\left(y, x_{3}\right) & =D_{u} \frac{H\left(x_{1}, x_{2}\right) H\left(x_{1}, x_{3}\right)}{A_{u}\left(x_{2}, x_{3}\right)}, \\
\sum_{y \in Y} A_{u}\left(x_{1}, y\right) H\left(x_{2}, y\right) H\left(x_{3}, y\right) & =D_{u} \frac{H\left(x_{2}, x_{1}\right) H\left(x_{3}, x_{1}\right)}{A_{u}\left(x_{2}, x_{3}\right)}, \\
\sum_{y \in Y} \frac{H\left(y, x_{1}\right) H\left(y, x_{2}\right)}{A_{u}\left(x_{3}, y\right)} & =D_{u} A_{u}\left(x_{1}, x_{2}\right) H\left(x_{3}, x_{1}\right) H\left(x_{3}, x_{2}\right), \\
\sum_{y \in Y} \frac{H\left(x_{1}, y\right) H\left(x_{2}, y\right)}{A_{u}\left(x_{3}, y\right)} & =D_{u} A_{u}\left(x_{1}, x_{2}\right) H\left(x_{1}, x_{3}\right) H\left(x_{2}, x_{3}\right),
\end{aligned}
$$

where

$$
D_{u}= \begin{cases}-u^{2}-u^{-2} & \text { if }|Y| \geq 2, \\ u^{2} & \text { if }|Y|=1 .\end{cases}
$$

We now prove Theorem 1.1. Since $A_{u}$ and $H$ are type II matrices, so are the matrices $T_{i j}=a^{\epsilon(i, j)} V_{i j}$ or $b^{\delta(i, j)} V_{i j}$. Thus, Lemma 2.1 implies that $W_{H, u, a}$ and $W_{H, u, b}^{\prime}$ are type II matrices.

We claim

$$
\sum_{y \in Y} \frac{V_{i_{1}, i_{0}}\left(x_{1}, y\right) V_{i_{2}, i_{0}}\left(x_{2}, y\right)}{V_{i_{3}, i_{0}}\left(x_{3}, y\right)}=D_{u} \frac{V_{i_{1}, i_{2}}\left(x_{1}, x_{2}\right)}{V_{i_{1}, i_{3}}\left(x_{1}, x_{3}\right) V_{i_{3}, i_{2}}\left(x_{3}, x_{2}\right)}
$$

for all $i_{1}, i_{2}, i_{3} \in \mathbb{Z}_{m}$ and $x_{1}, x_{2}, x_{3} \in Y$, where $i_{0}=i_{1}+i_{2}-i_{3} \bmod m$. Indeed, let $i_{1}, i_{2}, i_{3} \in \mathbb{Z}_{m}$. Then

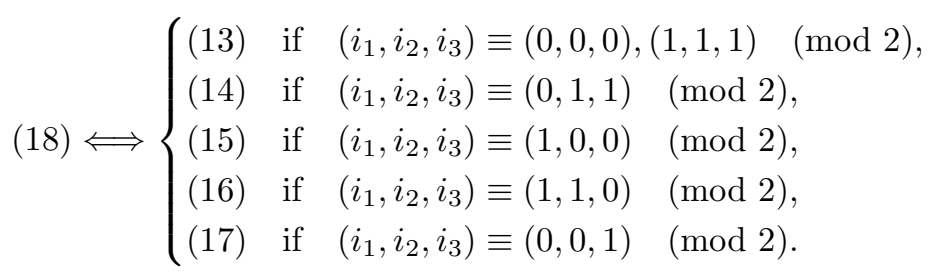

Moreover, when $\left(i_{1}, i_{2}, i_{3}\right) \equiv(1,0,1),(0,1,0)(\bmod 2)$, (18) is equivalent to (14), (15), respectively, with $x_{1}$ and $x_{2}$ switched. Therefore, (18) holds in all cases by Lemma 3.2

First, we show that $W_{H, u, a}$ and $W_{H, u, b}^{\prime}$ satisfy the condition (10). From Lemma 3.1 we have

$$
a^{\lambda_{\epsilon}\left(i_{1}, i_{2}, i_{3}, i_{0}\right)}=1 \quad \text { and } \quad b^{\lambda_{\delta}\left(i_{1}, i_{2}, i_{3}, i_{0}\right)}=1 .
$$

In view of (12), these imply

$$
c^{g\left(i_{1}, i_{0}\right)+g\left(i_{2}, i_{0}\right)-g\left(i_{3}, i_{0}\right)}=c^{g\left(i_{1}, i_{2}\right)-g\left(i_{1}, i_{3}\right)-g\left(i_{3}, i_{2}\right)},
$$


where $(c, g)=(a, \epsilon),(b, \delta)$. Combining (18) and (19), we obtain

$$
\begin{aligned}
& \sum_{y \in Y} \frac{c^{g\left(i_{1}, i_{0}\right)} V_{i_{1}, i_{0}}\left(x_{1}, y\right) c^{g\left(i_{2}, i_{0}\right)} V_{i_{2}, i_{0}}\left(x_{2}, y\right)}{c^{g\left(i_{3}, i_{0}\right)} V_{i_{3}, i_{0}}\left(x_{3}, y\right)} \\
& =D_{u} \frac{c^{g\left(i_{1}, i_{2}\right)} V_{i_{1}, i_{2}}\left(x_{1}, x_{2}\right)}{c^{g\left(i_{1}, i_{3}\right)} V_{i_{1}, i_{3}}\left(x_{1}, x_{3}\right) c^{g\left(i_{3}, i_{2}\right)} V_{i_{3}, i_{2}}\left(x_{3}, x_{2}\right)} .
\end{aligned}
$$

for all $i_{1}, i_{2}, i_{3} \in \mathbb{Z}_{m}$ and $x_{1}, x_{2}, x_{3} \in Y$. Thus (10) holds by setting $D=m D_{u}$. It follows from Lemma 2.2 that $W_{H, u, a}$ and $W_{H, u, b}^{\prime}$ satisfy the type III condition (8), and hence they are spin models. Since $\delta(i, j)=\delta(j, i), W_{H, u, b}^{\prime}$ is symmetric.

Finally, we show that $W_{H, u, a}$ has index $m$. Since $a^{2 m}=\eta$, we have $a^{\epsilon(i, j)-\epsilon(j, i)}=$ $a^{2 m(i-j)}=\eta^{i-j}$. So, $T_{i j}=\eta^{i-j} T_{j i}^{T}$ holds for all $i, j \in \mathbb{Z}_{m}$. From Lemma2.3, $W_{H, u, a}$ has index $m$. This completes the proof of Theorem 1.1

\section{Properties of Spin models in Theorem 1.1}

For a positive integer $r$, we let $u$ be a complex number satisfying (11).

Lemma 4.1. If $r \leq 4$, then $u$ is a root of unity. Otherwise, $|u| \neq 1$. If $r \geq 4$ or $r=1$, then $u^{4}>0$.

Proof. If $u$ is a root of unity and $r>1$, then $r=\left(u^{2}+u^{-2}\right)^{2} \leq|u|^{4}+2+|u|^{-4}=4$. It is easy to see that $u$ is indeed a root of unity if $r \leq 4$. If $r \geq 4$ or $r=1$, then we have $u^{4}>0$ from (11).

For a matrix $W \in \operatorname{Mat}_{X}\left(\mathbb{C}^{*}\right)$, we define

$$
E(W)=\left\{\frac{|W(x, y)|}{|W(x, x)|} \mid x, y \in X\right\} \subset \mathbb{R}_{>0} .
$$

Then

$$
E\left(W_{1} \otimes W_{2}\right)=E\left(W_{1}\right) E\left(W_{2}\right)
$$

holds for any matrices $W_{1}, W_{2}$ with nonzero entries.

For the remainder of this section, let $W_{H, u, a}, W_{H, u, b}^{\prime}$ be the spin models given in Theorem 1.1(i) and (ii), respectively. This means that $m$ is an even positive integer, $a$ is a primitive $2 m^{2}$-th root of unity, $b$ is an $m^{2}$-th root of unity, and $H$ is a Hadamard matrix of order $r$.

Lemma 4.2. We have

$$
E\left(W_{H, u, a}\right)=E\left(W_{H, u, b}^{\prime}\right)= \begin{cases}\left\{1,|u|^{-4},|u|^{-3}\right\} & \text { if } r>4, \\ \{1\} & \text { otherwise. }\end{cases}
$$

Proof. Immediate from Theorem 1.1 and Lemma 4.1

Lemma 4.3. (i) Suppose $r \geq 4$ or $r=1$. Then the entries of $W_{H, u, a}, W_{H, u, b}^{\prime}$ which have absolute value 1 are $2 m^{2}$-th roots of unity, $m^{2}$-th roots of unity, respectively. Moreover, $W_{H, u, a}$ contains a primitive $2 m^{2}$-th root of unity as one of its entries.

(ii) Suppose $r=2$, and put $\nu=\operatorname{LCM}\left(2 m^{2}, 16\right), \nu^{\prime}=\operatorname{LCM}\left(m^{2}, 16\right)$. Then the entries of $W_{H, u, a}, W_{H, u, b}^{\prime}$ are $\nu$-th roots of unity, $\nu^{\prime}$-th roots of unity, respectively. Moreover, $W_{H, u, a}$ contains a primitive $\nu$-th root of unity as one of its entries. 


\begin{tabular}{|c|c|c|l|l|c|}
\hline$W$ & index & size & $r$ & $\mu(W)$ & $E(W)$ \\
\hline \hline$W_{H, u, a}$ & $m$ & $m^{2} r$ & $r=1$ & $2 m^{2}$ & $\{1\}$ \\
& & & $r=2$ & $\mu(W) \mid \operatorname{LCM}\left(2 m^{2}, 16\right)$ & $\{1\}$ \\
& & & $r=4$ & $2 m^{2}$ & $\{1\}$ \\
& & & $r>4$ & $2 m^{2}$ & $\left\{1,|u|^{-4},|u|^{-3}\right\}$ \\
\hline$W_{H, u, b}^{\prime}$ & 1 & $m^{2} r$ & $r=1$ & $\mu(W) \mid m^{2}$ & $\{1\}$ \\
& & & $r=2$ & $\mu(W) \mid \operatorname{LCM}\left(m^{2}, 16\right)$ & $\{1\}$ \\
& & & $r=4$ & $\mu(W) \mid m^{2}$ & $\{1\}$ \\
& & & $r>4$ & $\mu(W) \mid m^{2}$ & $\left\{1,|u|^{-4},|u|^{-3}\right\}$ \\
\hline
\end{tabular}

TABLE 1. Summary of Properties

Proof. Firstly, suppose $r>4$. From Lemma 4.1, the entries of $W_{H, u, a}, W_{H, u, b}^{\prime}$ with absolute value 1 are

$$
\begin{aligned}
\pm a^{2 m\left(\ell-\ell^{\prime}\right)(i-j)+\epsilon(i, j)} & (i-j: \text { odd }) \\
\pm \eta^{\left(\ell-\ell^{\prime}\right)(i-j)} b^{\delta(i, j)} & (i-j: \text { odd })
\end{aligned}
$$

which are $2 m^{2}$-th roots of unity, $m^{2}$-th roots of unity, respectively. Putting $i=1$, $j=\ell=\ell^{\prime}=0$ in (21), we obtain $a^{1+m}$ which is a primitive $2 m^{2}$-th root of unity.

Next, suppose $r \leq 4$. Then the entries of $W_{H, u, a}, W_{H, u, b}^{\prime}$ are given by

$$
\begin{aligned}
v a^{2 m\left(\ell-\ell^{\prime}\right)(i-j)+\epsilon(i, j)} & \left(v \in\left\{u^{3},-u^{-1}, \pm 1\right\}\right), \\
v \eta^{\left(\ell-\ell^{\prime}\right)(i-j)} b^{\delta(i, j)} & \left(v \in\left\{u^{3},-u^{-1}, \pm 1\right\}\right),
\end{aligned}
$$

respectively, all of which are roots of unity.

If $r=4$ or 1 , then from (11), $u^{4}=1$. From (22), (23), the entries of $W_{H, u, a}$, $W_{H, u, b}^{\prime}$ are $2 m^{2}$-th roots of unity, $m^{2}$-th roots of unity, respectively. Putting $i=1$, $j=\ell=\ell^{\prime}=0$ in (22), we obtain $a^{1+m}$ which is a primitive $2 m^{2}$-th root of unity.

Finally, suppose $r=2$. Since $u$ is a primitive 16-root of unity by (11), the expressions in (22), (23) are $\nu$-th roots of unity, an $\nu^{\prime}$-th roots of unity, respectively. Putting $v=u^{3}, i=1, j=\ell=\ell^{\prime}=0$ in (22), we obtain $u^{3} a^{1+m}$ which is a primitive $\nu$-th root of unity.

For $S \in \operatorname{Mat}_{X}\left(\mathbb{C}^{*}\right)$, we denote by $\mu(S)$ the least common multiple of the orders of the entries of $S$ which have a finite order. If none of the entries of $S$ has a finite order, then we define $\mu(S)=\infty$. For a nonzero complex number $\zeta$, we denote by the same symbol $\mu(\zeta)$ the order of $\zeta$ if $\zeta$ has a finite order.

Lemma 4.4. Suppose $m \equiv 0(\bmod 4)$. Then for $W=W_{H, u, a}$ or $W=W_{H, u, b}^{\prime}$, we have $\mu(W) \mid 2 m^{2}$.

Proof. Immediate from Lemma 4.3.

In Table 1, we summarize the properties of $W=W_{H, u, a}, W_{H, u, b}^{\prime}$ obtained from Lemmas 4.1, 4.2, and 4.4 .

For $W \in \operatorname{Mat}_{X}\left(\mathbb{C}^{*}\right)$ and for a permutation $\sigma$ of $X$, we define $W^{\sigma}$ by $W^{\sigma}(\alpha, \beta)=$ $W(\sigma(\alpha), \sigma(\beta))$ for $\alpha, \beta \in X$. Observe that if $W$ is a spin model, then $W^{\sigma}$ is also a spin model. If $W$ is a spin model, then from (7), (8), $-W$ and $\pm \sqrt{-1} W$ are also spin models. Two spin models $W_{1}, W_{2}$ are said to be equivalent if $c W_{1}^{\sigma}=W_{2}$ for some permutation $\sigma$ of $X$ and a complex number $c$ with $c^{4}=1$. 
Two Hadamard matrices are said to be equivalent if one can be obtained from the other by negating rows and columns, or and permuting rows and columns.

Lemma 4.5. Let $H_{1}, H_{2} \in \operatorname{Mat}_{Y}\left(\mathbb{C}^{*}\right)$ be equivalent Hadamard matrices. Then $W_{H_{1}, u, a}$ is equivalent to $W_{H_{2}, u, a}$, and $W_{H_{1}, u, b}^{\prime}$ is equivalent to $W_{H_{2}, u, b}^{\prime}$.

Proof. Let $\left(W_{1}, W_{2}, c, g\right)=\left(W_{H_{1}, u, a}, W_{H_{2}, u, a}, a, \epsilon\right)$ or $\left(W_{H_{1}, u, b}^{\prime}, W_{H_{2}, u, b}^{\prime}, b, \delta\right)$.

If $\mathrm{H}_{2}$ is obtained by a permutation of columns of $H_{1}$, then there exists a permutation $\pi$ of $Y$ such that $H_{2}(x, \pi(y))=H_{1}(x, y)$ for all $x, y \in Y$. We define a permutation $\sigma$ of $X$ by

$$
\sigma((i, \ell, x))= \begin{cases}(i, \ell, \pi(x)) & \text { if } i \text { is odd } \\ (i, \ell, x) & \text { otherwise. }\end{cases}
$$

Then for $\alpha=(i, \ell, x), \beta=\left(j, \ell^{\prime}, y\right) \in X$,

$$
\begin{aligned}
W_{2}^{\sigma}(\alpha, \beta) & =W_{2}(\sigma(\alpha), \sigma(\beta)) \\
& = \begin{cases}c^{g(i, j)} S_{i j}\left(\ell, \ell^{\prime}\right) A_{u}(x, y) & \text { if } i \equiv j \equiv 0 \quad(\bmod 2), \\
c^{g(i, j)} S_{i j}\left(\ell, \ell^{\prime}\right) H_{2}(x, \pi(y)) & \text { if } i \equiv j+1 \equiv 0 \quad(\bmod 2), \\
c^{g(i, j)} S_{i j}\left(\ell, \ell^{\prime}\right) H_{2}^{T}(\pi(x), y) & \text { if } i+1 \equiv j \equiv 0 \quad(\bmod 2), \\
c^{g(i, j)} S_{i j}\left(\ell, \ell^{\prime}\right) A_{u}(\pi(x), \pi(y)) & \text { if } i \equiv j \equiv 1 \quad(\bmod 2)\end{cases} \\
= & \begin{cases}c^{g(i, j)} S_{i j}\left(\ell, \ell^{\prime}\right) A_{u}(x, y) & \text { if } i \equiv j \equiv 0 \quad(\bmod 2), \\
c^{g(i, j)} S_{i j}\left(\ell, \ell^{\prime}\right) H_{1}(x, y) & \text { if } i \equiv j+1 \equiv 0 \quad(\bmod 2), \\
c^{g(i, j)} S_{i j}\left(\ell, \ell^{\prime}\right) H_{1}^{T}(x, y) & \text { if } i+1 \equiv j \equiv 0 \quad(\bmod 2), \\
c^{g(i, j)} S_{i j}\left(\ell, \ell^{\prime}\right) A_{u}(x, y) & \text { if } i \equiv j \equiv 1 \quad(\bmod 2)\end{cases} \\
= & W_{1}(\alpha, \beta) .
\end{aligned}
$$

If $H_{2}$ is obtained by a permutation of rows of $H_{1}$, then there exists a permutation $\pi^{\prime}$ of $Y$ such that $H_{2}\left(\pi^{\prime}(x), y\right)=H_{1}(x, y)$ for all $x, y \in Y$. We define a permutation $\sigma^{\prime}$ of $X$ by

$$
\sigma^{\prime}((i, \ell, x))= \begin{cases}\left(i, \ell, \pi^{\prime}(x)\right) & \text { if } i \text { is even } \\ (i, \ell, x) & \text { otherwise. }\end{cases}
$$

Similar calculation shows $W_{2}^{\sigma^{\prime}}(\alpha, \beta)=W_{1}(\alpha, \beta)$.

If $H_{2}$ is obtained by negating a column $y_{1}$ of $H_{1}$, then $H_{2}\left(x, y_{1}\right)=-H_{1}\left(x, y_{1}\right)$, $H_{2}(x, y)=H_{1}(x, y)$ for all $x \in Y$ and $y \in Y-\left\{y_{1}\right\}$. We define a permutation $\rho$ of $X$ by

$$
\rho((i, \ell, x))= \begin{cases}\left(i, \ell+\delta_{x, y_{1}} \frac{m}{2}, x\right) & \text { if } i \text { is odd } \\ (i, \ell, x) & \text { otherwise }\end{cases}
$$

Note that $S_{i j}\left(\ell, \ell^{\prime}\right)=(-1)^{i-j} S_{i j}\left(\ell+\frac{m}{2}, \ell^{\prime}\right)=(-1)^{i-j} S_{i j}\left(\ell, \ell^{\prime}+\frac{m}{2}\right)$. Thus for $\alpha=(i, \ell, x), \beta=\left(j, \ell^{\prime}, y\right) \in X$,

$$
\begin{aligned}
& W_{2}^{\rho}(\alpha, \beta) \\
& =W_{2}(\rho(\alpha), \rho(\beta))
\end{aligned}
$$




$$
\begin{aligned}
& = \begin{cases}c^{g(i, j)} S_{i j}\left(\ell, \ell^{\prime}\right) A_{u}(x, y) & \text { if } i \equiv j \equiv 0 \quad(\bmod 2), \\
c^{g(i, j)} S_{i j}\left(\ell, \ell^{\prime}+\delta_{y, y_{1}} \frac{m}{2}\right) H_{2}(x, y) & \text { if } i \equiv j+1 \equiv 0 \quad(\bmod 2), \\
c^{g(i, j)} S_{i j}\left(\ell+\delta_{x, y_{1}} \frac{m}{2}, \ell^{\prime}\right) H_{2}^{T}(x, y) & \text { if } i+1 \equiv j \equiv 0 \quad(\bmod 2), \\
c^{g(i, j)} S_{i j}\left(\ell+\delta_{x, y_{1}} \frac{m}{2}, \ell^{\prime}+\delta_{y, y_{1}} \frac{m}{2}\right) A_{u}(x, y) & \text { if } i \equiv j \equiv 1 \quad(\bmod 2)\end{cases} \\
& = \begin{cases}c^{g(i, j)} S_{i j}\left(\ell, \ell^{\prime}\right) A_{u}(x, y) & \text { if } i \equiv j \equiv 0 \quad(\bmod 2), \\
(-1)^{\delta_{y, y_{1}}} c^{g(i, j)} S_{i j}\left(\ell, \ell^{\prime}\right) H_{2}(x, y) & \text { if } i \equiv j+1 \equiv 0 \quad(\bmod 2), \\
(-1)^{\delta_{x, y_{1}}} c^{g(i, j)} S_{i j}\left(\ell, \ell^{\prime}\right) H_{2}^{T}(x, y) & \text { if } i+1 \equiv j \equiv 0 \quad(\bmod 2), \\
c^{g(i, j)} S_{i j}\left(\ell, \ell^{\prime}\right) A_{u}(x, y) & \text { if } i \equiv j \equiv 1 \quad(\bmod 2)\end{cases} \\
& =\left\{\begin{array}{lll}
c^{g(i, j)} S_{i j}\left(\ell, \ell^{\prime}\right) A_{u}(x, y) & \text { if } i \equiv j \equiv 0 \quad(\bmod 2), \\
c^{g(i, j)} S_{i j}\left(\ell, \ell^{\prime}\right) H_{1}(x, y) & \text { if } i \equiv j+1 \equiv 0 \quad(\bmod 2), \\
c^{g(i, j)} S_{i j}\left(\ell, \ell^{\prime}\right) H_{1}^{T}(x, y) & \text { if } i+1 \equiv j \equiv 0 \quad(\bmod 2), \\
c^{g(i, j)} S_{i j}\left(\ell, \ell^{\prime}\right) A_{u}(x, y) & \text { if } i \equiv j \equiv 1 \quad(\bmod 2)
\end{array}\right. \\
& =W_{1}(\alpha, \beta) .
\end{aligned}
$$

If $H_{2}$ is obtained by negating a row $x_{1}$ of $H_{1}$, then $H_{2}\left(x_{1}, y\right)=-H_{1}\left(x_{1}, y\right)$, $H_{2}(x, y)=H_{1}(x, y)$ for all $x \in Y-\left\{x_{1}\right\}$ and $y \in Y$. We define a permutation $\rho^{\prime}$ of $X$ by

$$
\rho^{\prime}((i, \ell, x))= \begin{cases}\left(i, \ell+\delta_{x, x_{1}} \frac{m}{2}, x\right) & \text { if } i \text { is even } \\ (i, \ell, x) & \text { otherwise. }\end{cases}
$$

Similar calculation shows $W_{2}^{\rho^{\prime}}(\alpha, \beta)=W_{1}(\alpha, \beta)$.

\section{Decomposability}

Lemma 5.1. Let $S_{1}, S_{2}$ be finite subsets of positive real numbers. Suppose $1 \in$ $S_{1} \cap S_{2}$ and $\left|S_{1} S_{2}\right|=3$. Then

$$
\left(\left|S_{1}\right|,\left|S_{2}\right|\right) \in\{(2,2),(1,3),(3,1)\} .
$$

If $\left|S_{1}\right|=\left|S_{2}\right|=2$, then $S_{1} S_{2}=\left\{1, a, a^{2}\right\}$ or $\left\{1, a, a^{-1}\right\}$ for some positive real number $a \neq 1$.

Proof. By way of contradiction, we prove that if $\left|S_{1}\right| \geq 3$ and $\left|S_{2}\right| \geq 2$ then $\left|S_{1} S_{2}\right|>$ 3. Since $S_{1} \cup S_{2} \subset S_{1} S_{2}$, we obtain $S_{2} \subset S_{1}=S_{1} S_{2}$. Let $S_{1}=\{1, \lambda, \mu\}(\lambda, \mu \neq$ $1, \lambda \neq \mu)$. Then we may put $S_{2}=\{1, \lambda\}$ without loss of generality. Then we have $\lambda^{2} \in S_{1} S_{2}=S_{1}$, so $\mu=\lambda^{2}$ and $S_{1} S_{2}=\left\{1, \lambda, \lambda^{2}, \lambda^{3}\right\}$. This implies $\left|S_{1} S_{2}\right|=4$, a contradiction.

Suppose $\left|S_{1}\right|=\left|S_{2}\right|=2$. Then $S_{1}=\{1, a\}, S_{2}=\{1, b\}$ for some $a, b \neq 1$. Then $\left|S_{1} S_{2}\right|=3$ implies $a=b$ or $a=b^{-1}$.

Lemma 5.2. Let $A \in \operatorname{Mat}_{Z_{1}}\left(\mathbb{C}^{*}\right)$ be a matrix all of whose entries are roots of unity. Let $B \in \operatorname{Mat}_{Z_{2}}\left(\mathbb{C}^{*}\right)$ be a matrix which satisfies $\mu(B)<\infty$. Then $\mu(A \otimes B)$ is a divisor of $\operatorname{LCM}(\mu(A), \mu(B))$.

Proof. Let $Z_{2}^{\prime}=\left\{\left(x_{2}, y_{2}\right) \in Z_{2} \times Z_{2} \mid o\left(B\left(x_{2}, y_{2}\right)\right)<\infty\right\}$. Then

$$
\mu(A \otimes B)=\operatorname{LCM}\left(\left\{o\left(A\left(x_{1}, y_{1}\right) B\left(x_{2}, y_{2}\right)\right) \mid x_{1}, y_{1} \in Z_{1},\left(x_{2}, y_{2}\right) \in Z_{2}^{\prime}\right\}\right),
$$

which is a divisor of $\operatorname{LCM}(\mu(A), \mu(B))$. 
Some examples of spin models are listed in Section 1, i.e., Potts model, nonsymmetric Hadamard models, and Hadamard models. We remark that non-symmetric Hadamard models and Hadamard models are special cases of spin models given in Theorem 1.1(i), (ii), respectively. In addition to these examples, the following spin models are known.

Spin models on finite abelian groups. Bannai-Bannai-Jaeger 3] gives solutions to modular invariance equation for finite abelian groups, and every solution gives a spin model. Let $U$ be a finite abelian group, and $e=\exp (U)$ denote the exponent of $U$. Let $\left\{\chi_{a} \mid a \in U\right\}$ be the set of characters of $U$ with indices chosen so that $\chi_{a}(b)=\chi_{b}(a)$ for all $a, b \in U$. Let $U=U_{1} \oplus \cdots \oplus U_{h}$ be a decomposition of $U$ into a direct sum of cyclic groups $U_{1}, U_{2}, \ldots, U_{h}$. For each $i \in\{1,2, \ldots, h\}$ let $a_{i}$ be a generator and $n_{i}$ be the order of the cyclic group $U_{i}$. For each $x \in U$, we define the matrix $A_{x} \in \operatorname{Mat}_{U}(\mathbb{C})$ by

$$
A_{x}(\alpha, \beta)=\delta_{x, \beta-\alpha} \quad(\alpha, \beta \in U) .
$$

For any $x=\sum_{i=1}^{h} x_{i} a_{i}\left(0 \leq x_{i}<n_{i}\right)$, let

$$
t_{x}=t_{0} \prod_{i=1}^{h} \eta_{i}^{x_{i}} \chi_{a_{i}}\left(a_{i}\right)^{\frac{x_{i}\left(x_{i}-1\right)}{2}} \prod_{1 \leq \ell<k \leq h} \chi_{a_{\ell}}\left(a_{k}\right)^{x_{\ell} x_{k}},
$$

where $\eta_{i}^{n_{i}}=\chi_{a_{i}}\left(a_{i}\right)^{-\frac{n_{i}\left(n_{i}-1\right)}{2}}$ and

$$
t_{0}^{2}=D^{-1} \sum_{x \in U} \prod_{j=1}^{h} \eta_{j}^{-x_{j}} \chi_{a_{j}}\left(a_{j}\right)^{-\frac{x_{j}\left(x_{j}-1\right)}{2}} \prod_{1 \leq \ell<k \leq h} \chi_{a_{\ell}}\left(a_{k}\right)^{-x_{\ell} x_{k}}
$$

where $D^{2}=|U|$. Let $\theta_{x}=t_{x} / t_{0}$ for any $x \in U$. Then, for any $x \in U, \theta_{x}$ is a root of unity and $\theta_{x}^{2 e}=1$. Especially, we get

$$
\theta_{x}^{2|U|}=1
$$

The matrix

$$
W=\sum_{x \in U} t_{x} A_{x}
$$

is a spin model.

Jaeger's Higman-Sims model. In [10], F. Jaeger constructed a spin model $W_{J}$ on the Higman-Sims graph of size 100 . We denote by $A$ the adjacency matrix of the Higman-Sims graph. We put $W_{J}=-\tau^{5} I-\tau A+\tau^{-1}(J-A-I)$, where $\tau$ satisfies $\tau^{2}+\tau^{-2}=3$. Then $W_{J}$ is a symmetric spin model.

Now every known spin model belongs to one of the following five families:

(a) $A_{u}$ : Potts model of size $r \geq 2$. If $r=2$, then $\mu\left(A_{u}\right)=16$. If $r=4$, then $\mu\left(A_{u}\right)=2$ or 4 . If $r=2,4$, then $E\left(A_{u}\right)=\{1\}$. If $r>4$, then $E\left(A_{u}\right)=\left\{1,|u|^{-4}\right\}$, and hence $\left|E\left(A_{u}\right)\right|=2$.

(b) $W_{U}$ : spin model on a finite abelian group $U$. We have various kinds of indices and $E\left(W_{U}\right)=\{1\}$.

(c) $W_{J}$ : Jaeger's Higman-Sims model of size 100. We have $E\left(W_{J}\right)=\left\{1, \tau^{-4}, \tau^{-6}\right\}$ with $\tau^{2}+\tau^{-2}=3$. and hence $\left|E\left(W_{J}\right)\right|=3$.

(d) $W_{H, u, a}$ : spin models given in Theorem 1.1)(i).

(e) $W_{H, u, b}^{\prime}$ : spin models given in Theorem 1.1(ii). 
By way of contradiction, we now give a proof of Theorem 1.3. Let $H$ be a Hadamard matrix of order $r>4$. Let $s$ be a positive integer and $a$ a primitive $2^{2 s+1}$-th root of unity. For the remainder of this section, we denote by $W$ the spin model $W_{H, u, a}$ given in Theorem 1.1 (i) of index $2^{s}$. By Lemma 4.2 we obtain

$$
E(W)=\left\{1,|u|^{-4},|u|^{-3}\right\} .
$$

We assume that

$$
W=W_{1} \otimes W_{2} \otimes \cdots \otimes W_{v}
$$

where each of $W_{1}, W_{2}, \ldots, W_{v}$ is a known spin model listed in (a)-(e) and their sizes are not equal to 1 . Since $|E(W)|=3$ from (28), using Lemma 5.1] we may assume without loss of generality

$$
\left(\left|E\left(W_{1}\right)\right|,\left|E\left(W_{2}\right)\right|, \ldots,\left|E\left(W_{v}\right)\right|\right)=(1, \ldots, 1,2,2) \text { or }(1, \ldots, 1,3) .
$$

A known spin model $W^{\prime}$ with $\left|E\left(W^{\prime}\right)\right|=1$ belongs to the family (b) or to the families (a), (d) and (e) with $r \leq 4$. Therefore, (29) can be reduced to the following cases:

$$
\begin{aligned}
& W=W_{1} \otimes W_{2} \otimes W_{3} \text { with } E\left(W_{1}\right)=\{1\},\left|E\left(W_{2}\right)\right|=\left|E\left(W_{3}\right)\right|=2, \\
& W=W_{1} \otimes W_{2} \text { with } E\left(W_{1}\right)=\{1\},\left|E\left(W_{2}\right)\right|=3,
\end{aligned}
$$

where in (30), (31), $W_{1}$ is a tensor product of spin models on finite abelian groups and spin models in the families (a), (d) and (e) with $r \leq 4$. Note that $W_{1}$ could possibly be of size 1 in (30).

First, we treat the case (30). Then Lemma 5.1 implies $E\left(W_{2} \otimes W_{3}\right)=\left\{1, \beta, \beta^{2}\right\}$, or $\left\{1, \beta, \beta^{-1}\right\}$ for some $\beta$. On the other hand, $E\left(W_{2} \otimes W_{3}\right)=E\left(W_{1}\right) E\left(W_{2} \otimes W_{3}\right)=$ $E(W)=\left\{1,|u|^{-4},|u|^{-3}\right\}$ by (28). This is a contradiction.

Next, we treat the case (31). We have $E\left(W_{2}\right)=E\left(W_{1}\right) E\left(W_{2}\right)=E(W)=$ $\left\{1,|u|^{-4},|u|^{-3}\right\}$ from (28). Since $\left\{1,|u|^{-4},|u|^{-3}\right\} \neq\left\{1, \tau^{-4}, \tau^{-6}\right\}, W_{2}$ cannot be the spin model (c). Therefore, $W_{2}$ belongs to the family (d) or (e). This means $W_{2}=W_{H^{\prime}, u^{\prime}, a^{\prime}}$ or $W_{2}=W_{H^{\prime}, u^{\prime}, b^{\prime}}^{\prime}$, where $H^{\prime}$ is a Hadamard matrix of order $r^{\prime}=\left(u^{\prime 2}+u^{\prime-2}\right)^{2}$. Since $\left|E\left(W_{2}\right)\right|=3$, Lemma 4.2 implies $r^{\prime}>4$ and $E\left(W_{2}\right)=$ $\left\{1,\left|u^{\prime}\right|^{-4},\left|u^{\prime}\right|^{-3}\right\}$. Then we have $\left|u^{\prime}\right|=|u|$, as $E(W)=E\left(W_{2}\right)$. Now the second part of Lemma 4.1 implies $u^{4}>0$ and $u^{\prime 4}>0$, hence

$$
u^{4}=u^{4} \text {, }
$$

and further $r=r^{\prime}$ by (11). Therefore the size of $W_{2}$ is $2^{2 s^{\prime}} r$ for some integer $s^{\prime}$ with $0<s^{\prime}<s$, and the size of $W_{1}$ is $2^{2\left(s-s^{\prime}\right)}$. In particular, we obtain $s>1$.

Since the tensor product of spin models on finite abelian groups is also a spin model on a finite abelian group, we may suppose that

$$
W_{1}=W_{11} \otimes W_{12} \otimes W_{13},
$$

where $W_{11}$ is a spin model on a finite abelian group $U, W_{12}$ is a tensor product of spin models in the family (a) with $r \leq 4$, and $W_{13}$ is a tensor product of spin models in the families (d) and (e) with $r \leq 4$.

We put $|U|=2^{n_{1}}$. Since the size $2^{n_{1}}$ of $W_{11}$ cannot exceed that of $W_{1}$, we have $n_{1} \leq 2\left(s-s^{\prime}\right)$. Then the size of $W_{12} \otimes W_{13}$ is $2^{2\left(s-s^{\prime}\right)-n_{1}}$. The diagonal entry of $W_{11}$ is a complex number $t_{0}$ given by (25). The diagonal entries of $W_{12}, W_{13}$ are 16-th roots of unity. We denote by $\kappa_{2}, \kappa_{3}$ the diagonal entries of $W_{12}, W_{13}$, respectively. Comparing the diagonal entries of (33), we have $u^{3}=t_{0} \kappa_{2} \kappa_{3} u^{\prime 3}$, thus

$$
W=\left(t_{0}^{-1} W_{11}\right) \otimes\left(\kappa_{2}^{-1} W_{12}\right) \otimes\left(\kappa_{3}^{-1} W_{13}\right) \otimes\left(u^{3} u^{\prime-3} W_{2}\right) .
$$


From (26), we have

$$
\mu\left(t_{0}^{-1} W_{11}\right) \mid 2^{n_{1}+1} .
$$

From (a), we have

$$
\mu\left(\kappa_{2}^{-1} W_{12}\right) \mid 2^{4}
$$

From (a) and Lemma 4.4, we have

$$
\mu\left(\kappa_{3}^{-1} W_{13}\right) \mid 2^{2\left(s-s^{\prime}\right)-n_{1}+1} .
$$

Since $W_{2}$ is a spin model belonging to the family (d) or (e), Lemma 4.3 and (32) imply

$$
\mu\left(u^{3} u^{\prime-3} W_{2}\right) \mid 2^{2 s^{\prime}+1} .
$$

From (34)-(38) and Lemma 5.2 we have

$$
\mu(W) \mid \operatorname{LCM}\left(2^{n_{1}+1}, 2^{4}, 2^{2\left(s-s^{\prime}\right)-n_{1}+1}, 2^{2 s^{\prime}+1}\right) .
$$

Since $n_{1}<2 s$, we have $\max \left(n_{1}+1,4,2\left(s-s^{\prime}\right)-n_{1}+1,2 s^{\prime}+1\right) \leq 2 s$. This implies $\mu(W) \mid 2^{2 s}$, which contradicts Lemma 4.3 (i).

\section{Spin models in Theorem 1.1 with $r \leq 4$}

In this section, we treat the case of $r \leq 4$ in Theorem 1.3. We show that if $r=1,4$ in Theorem 1.3, then $W_{H, u, a}$ is not new.

If $r=4$ in Theorem 1.1(i), then $W_{H, u, a}$ is a tensor product of a Hadamard matrix of order 4 and $W_{(1), u, a}$. Indeed, up to equivalence, there is a unique Hadamard matrix of order $r=4$. By Lemma 4.5, we may assume without loss of generality

$$
H=\left(\begin{array}{cccc}
1 & -1 & -1 & -1 \\
-1 & 1 & -1 & -1 \\
-1 & -1 & 1 & -1 \\
-1 & -1 & -1 & 1
\end{array}\right)
$$

Then $A_{u}=u^{3} H$ with $\left(u^{2}+u^{-2}\right)^{2}=4$. Therefore we have $W_{H, u, a}=H \otimes W_{(1), u, a}$. Similarly, a spin model $W_{H, u, b}^{\prime}$ in Theorem 1.1 (ii) can be decomposed as $H \otimes$ $W_{(1), u, b}^{\prime}$.

Lemma 6.1. Let $m \equiv 0(\bmod 4)$. Let $W_{(1), u, a}$ be a spin model given in Theorem 1.1 of index $m$, where $u^{4}=1$ and $a$ is a primitive $2 m^{2}$-th root of unity. Then $W_{(1), u, a}$ is equivalent to $W_{(1), 1, a u^{3}}$.

Proof. First we assume that $u=-1$. Then $a^{\epsilon(i, j)}(-1)^{i-j-1}=-(-a)^{\epsilon(i, j)}$ holds for all $i, j \in \mathbb{Z}_{m^{2}}$. From this, we have $W_{(1),-1, a}=-W_{(1), 1,-a}$. Therefore $W_{(1),-1, a}$ is equivalent to $W_{(1), 1,-a}$.

Next we assume that $u^{2}=-1$. Since $m \equiv 0(\bmod 4)$, we have

$$
u\left(a u^{3}\right)^{\epsilon(i, j)}= \begin{cases}a^{\epsilon(i, j)} u & \text { if } i-j \text { is even, } \\ a^{\epsilon(i, j)} & \text { if } i-j \text { is odd. }\end{cases}
$$

From this, we have $u W_{(1), 1, a u^{3}}=W_{(1), u, a}$. Therefore $W_{(1), u, a}$ is equivalent to $W_{(1), 1, a u^{3}}$.

Lemma 6.2. Let $m$ be even, and $\xi$ be a primitive $2 m^{2}$-th root of unity. Then we have

$$
\sum_{x=0}^{m^{2}-1} \xi^{-x(x-m)}=m .
$$


Proof. If (39) holds for $\xi=\exp \left(2 \pi \sqrt{-1} /\left(2 m^{2}\right)\right)$, then by considering the action of the Galois group, we see that (39) holds for any primitive $2 m^{2}$-th root of unity $\xi$. Therefore we may assune $\xi=\exp \left(2 \pi \sqrt{-1} /\left(2 m^{2}\right)\right)$ without loss of generality. Since $m$ is even, we may write $m=2 k$. Then

$$
\begin{aligned}
\sum_{x=0}^{m^{2}-1} \xi^{-x(x-m)} & =\sum_{x=0}^{m^{2}-1} \xi^{-\left((x-k)^{2}-k^{2}\right)} \\
& =\xi^{k^{2}} \sum_{x=0}^{m^{2}-1} \xi^{-(x-k)^{2}} \\
& =\frac{\xi^{k^{2}}}{2} \sum_{x=0}^{m^{2}-1}\left(\xi^{-(x-k)^{2}}+\xi^{-\left(x-k+m^{2}\right)^{2}}\right) \\
& =\frac{\exp (\pi \sqrt{-1} / 4)}{2} \sum_{x=0}^{2 m^{2}-1} \xi^{-(x-k)^{2}} \\
& =\frac{1+\sqrt{-1}}{2 \sqrt{2}} \sum_{x=0}^{2 m^{2}-1} \xi^{-x^{2}} .
\end{aligned}
$$

Now the result follows from [16, Theorem 99].

Of particular interest among spin models on finite abelian groups are spin models on finite cyclic groups. The spin model defined below is a special case of spin models on finite cyclic groups constructed by [1]. Let $m$ be even, and $a$ be a primitive $2 m^{2}$ th root of unity. We restrict (24) and (25) to $\mathbb{Z}_{m^{2}}$, that is, $h=1$. In (24) and (25), we put $\eta_{1}=a^{-m+1}, \chi_{a_{1}}\left(a_{1}\right)=a^{2}$. Then (24) and (25) become

$$
\begin{aligned}
t_{x} & =t_{0} a^{x(x-m)} \quad\left(x \in \mathbb{Z}_{m^{2}}\right), \\
t_{0}^{2} & =m^{-1} \sum_{x=0}^{m^{2}-1} a^{-x(x-m)}=1,
\end{aligned}
$$

respectively, where we used Lemma 6.2 in (41). Thus we may take $t_{0}=1$. Then the matrix $W$ given in (27) has entries

$$
W(\alpha, \beta)=a^{(\beta-\alpha)(\beta-\alpha-m)} \quad\left(\alpha, \beta \in \mathbb{Z}_{m^{2}}\right) .
$$

We note that this spin model $W$ on $\mathbb{Z}_{m^{2}}$ was constructed originally in [2, Theorem 2].

Proposition 6.3. Let $m \equiv 0(\bmod 4)$. Let $W_{(1), u, a}$ be a spin model given in Theorem 1.1(i) of index $m$, where $u^{4}=1$ and a is a primitive $2 m^{2}$-th root of unity. Then $W_{(1), u, a}$ is equivalent to $W$ defined in (42).

Proof. From Lemma 6.1] it is sufficient to prove that $W_{(1), 1, a u^{3}}$ is equivalent to $W$. By assumption, $m=4 k$ for some positive integer $k$. Since $a^{8 k^{2}}$ is a primitive 4th root of unity, there exists $t \in \mathbb{Z}_{4}$ such that $u^{3}=a^{8 k^{2} t}$. We define a bijection $\psi: \mathbb{Z}_{m}^{2} \rightarrow \mathbb{Z}_{m^{2}}$ by

$$
\psi(i, \ell)=\left(4 k^{2} t+1\right) i+4 k \ell
$$


for $(i, \ell) \in \mathbb{Z}_{m}^{2}$. Then for all $i, j, \ell, \ell^{\prime} \in \mathbb{Z}_{m}$,

$$
\begin{aligned}
& \left(\psi\left(j, \ell^{\prime}\right)-\psi(i, \ell)\right)\left(\psi\left(j, \ell^{\prime}\right)-\psi(i, \ell)-m\right) \\
& =\left(\left(4 k^{2} t+1\right)(j-i)+4 k\left(\ell^{\prime}-\ell\right)\right)\left(\left(4 k^{2} t+1\right)(j-i)+4 k\left(\ell^{\prime}-\ell\right)-4 k\right) \\
& =\left(8 k^{2} t+1\right)\left(8 k\left(\ell-\ell^{\prime}\right)(i-j)+(i-j)^{2}+4 k(i-j)\right) \\
& \quad+32 k^{2}\left(-k t(j-i)\left(l^{\prime}-l\right)+\frac{k t(j-i)(k t(j-i)+1)}{2}\right. \\
& \left.\quad+\frac{\left(l^{\prime}-l\right)\left(l^{\prime}-l-1\right)}{2}\right) \\
& \quad\left(8 k^{2} t+1\right)\left(8 k\left(\ell-\ell^{\prime}\right)(i-j)+(i-j)^{2}+4 k(i-j)\right) \quad\left(\bmod 32 k^{2}\right) .
\end{aligned}
$$

Thus

$$
\begin{aligned}
W\left(\psi(i, \ell), \psi\left(j, \ell^{\prime}\right)\right) & =a^{\left(\psi\left(j, \ell^{\prime}\right)-\psi(i, \ell)\right)\left(\psi\left(j, \ell^{\prime}\right)-\psi(i, \ell)-m\right)} \\
& =a^{\left(8 k^{2} t+1\right)\left(8 k\left(\ell-\ell^{\prime}\right)(i-j)+(i-j)^{2}+4 k(i-j)\right)} \\
& =\left(a u^{3}\right)^{2 m\left(\ell-\ell^{\prime}\right)(i-j)+(i-j)^{2}+m(i-j)} \\
& =W_{(1), 1, a u^{3}}\left((i, \ell, 1),\left(j, \ell^{\prime}, 1\right)\right),
\end{aligned}
$$

and we conclude that $W$ is equivalent to $W_{(1), 1, a u^{3}}$.

To conclude the paper, we note that the decomposability and identification with known spin models are yet to be determined for the following cases.

(1) $W_{H, u, a}: r=1, m \equiv 2(\bmod 4)$,

(2) $W_{H, u, b}^{\prime}: r=1$,

(3) $W_{H, u, a}$ and $W_{H, u, b}^{\prime}: r=2$,

(4) $W_{H, u, a}$ and $W_{H, u, b}^{\prime}: r>4$ and $m$ is not a power of 2 .

\section{REFERENCES}

[1] E. Bannai and Et. Bannai, Spin models on finite cyclic groups, J. Algebraic Combin. 3 (1994), 243-259.

[2] E. Bannai and Et. Bannai, Generalized generalized spin models (four-weight spin models), Pacific J. Math. 170 (1995), 1-16.

[3] E. Bannai, Et. Bannai and F. Jaeger On spin models, modular invariance, and duality, J. Algebraic Combin. 6 (1997), 203-228.

[4] E. Bannai and T. Ito, Algebraic Combinatorics I, Benjamin/Cummings, Menlo Park, 1984.

[5] Et. Bannai and A. Munemasa, Duality maps of finite abelian groups and their applications to spin models, J. Algebraic Combin. 8 (1998), 223-233.

[6] C. Bracken and G. McGuire, Characterization of SDP designs that yield certain spin models, Des. Codes Cryptogr. 36 (2005), 45-52.

[7] C. Bracken and G. McGuire, On quasi-3 designs and spin models, Discrete Math. 294 (2005), $21-24$.

[8] C. Godsil and A. Roy, Equiangular lines, mutually unbiased bases, and spin models, European J. Combin. 30 (2009), 246-262.

[9] T. Ikuta and K. Nomura, General form of non-symmetric spin models, J. Algebraic Combin. 12 (2000), 59-72.

[10] F. Jaeger, Strongly regular graphs and spin models for the Kauffman polynomial, Geom. Dedicata 44 (1992), 23-52.

[11] F. Jaeger, M. Matsumoto, and K. Nomura, Bose-Mesner algebras related to type II matrices and spin models, J. Algebraic Combin. 8 (1998), 39-72.

[12] F. Jaeger and K. Nomura, Symmetric versus non-symmetric spin models for link invariants, J. Algebraic Combin. 10 (1999), 241-278. 
[13] V.F.R. Jones, On knot invariants related to some statistical mechanical models, Pacific J. Math. 137 (1989), 311-336.

[14] K. Kawagoe, A. Munemasa, and Y. Watatani, Generalized spin models, J. Knot Theory Ramifications 3 (1994), 465-475.

[15] P. Manches and S. Ceroi, Spin models, association schemes and the Nakanishi-Montesinos Conjecture, European J. Combin. 23 (2002), 833-844.

[16] T. Nagell, Introduction to Number Theory, Almqvist and Wiksell, Stockholm, and John Wiley and Sons, New York (1951) (Reprinted by Chelsea Publishing Company, New York.).

[17] K. Nomura, Spin models constructed from Hadamard matrices, J. Combin. Theory Ser. A 68 (1994), 251-261.

[18] K. Nomura, An algebra associated with a spin model, J. Algebraic Combin. 6 (1997), 53-58.

[19] K. Nomura, Spin models of index 2 and Hadmard models, J. Algebraic Combin. 17 (2003), $5-17$.

Faculty of Law, Kobe Gakuin University, Minatojima, ChuO-Ku, Kobe, 650-8586 Japan

E-mail address: ikuta@law.kobegakuin.ac.jp

Graduate School of Information Sciences, Tohoku University, Sendai, 980-8579 Japan

E-mail address: munemasa@math.is.tohoku.ac.jp 